\title{
Multiple-therapy-resistant major depressive disorder: a clinically important concept
}

R. H. McAllister-Williams, D. M. B. Christmas, A. J. Cleare, A. Currie, J. Gledhill, L. Insole, A. L. Malizia, M. McGeever, R. Morriss, L. J. Robinson, M. Scott, P. R. A. Stokes, P. S. Talbot and A. H. Young

\section{Summary}

Many novel therapeutic options for depression exist that are either not mentioned in clinical guidelines or recommended only for use in highly specialist services. The challenge faced by clinicians is when it might be appropriate to consider such 'nonstandard' interventions. This analysis proposes a framework to aid this decision.

\section{Declaration of interest}

In the past 3 years R.H.M.W. has received support for research, expenses to attend conferences and fees for lecturing and consultancy work (including attending advisory boards) from various pharmaceutical companies including Astra Zeneca, Cyberonics, Eli Lilly, Janssen, LivaNova, Lundbeck,

MyTomorrows, Otsuka, Pfizer, Roche, Servier, SPIMACO and Sunovion. D.M.B.C. has received fees from LivaNova for attending an advisory board. In the past 3 years A.J.C. has received fees for lecturing from Astra Zeneca and Lundbeck; fees for consulting from LivaNova, Janssen and Allergan; and research grant support from Lundbeck.

In the past 3 years A.C. has received fees for lecturing from pharmaceutical companies namely Lundbeck and Sunovion. In the past 3 years A.L.M. has received support for attending seminars and fees for consultancy work (including advisory board) from Medtronic Inc and LivaNova. R.M. holds joint research grants with a number of digital companies that investigate devices for depression including Alpha-stim, Big White Wall, P1vital, Intel, Johnson and Johnson and Lundbeck through his mindTech and CLAHRC EM roles. M.S. is an associate at Blueriver Consulting providing intelligence to NHS organisations, pharmaceutical and devices companies. He has received honoraria for presentations and advisory boards with Lundbeck, Eli Lilly, URGO, AstraZeneca, Phillips and Sanofi and holds shares in Johnson and Johnson. In the past 3 years P.R.A.S. has received support for research, expenses to attend conferences and fees for lecturing and consultancy work (including attending an advisory board) from life sciences companies including corcept Therapeutics, Indivior and LivaNova. In the past 3 years P.S.T. has received consultancy fees as an advisory board member from the following companies: Galen Limited, Sunovion Pharmaceuticals Europe Ltd, myTomorrows and LivaNova.

A.H.Y. has undertaken paid lectures and advisory boards for all major pharmaceutical companies with drugs used in affective and related disorders and LivaNova. He has received funding for investigator initiated studies from AstraZeneca, Eli Lilly, Lundbeck and wyeth.

\section{Copyright and usage}

(c) The Royal College of Psychiatrists 2018.
There are numerous effective psychological therapies and drug treatments for the management of major depressive disorder (MDD) and guidelines for their use (for example those from the UK's National Institute for Health and Care Excellence (NICE) ${ }^{1}$ or British Association for Psychopharmacology $\left.(\mathrm{BAP})^{2}\right)$. However, a major challenge is improving the management of patients with MDD who do not experience full or sustained remission of symptoms with standard, guideline-recommended treatments or who cannot tolerate them. A major issue is that many of the recommended treatments have similar mechanisms of action and in naturalistic studies there appears to be minimal difference in effectiveness between them. ${ }^{1,2}$ In addition, duration of depression is associated with worse outcomes, ${ }^{3}$ including social breakdown such as loss of job or relationship, ${ }^{4}$ which in themselves can become barriers to recovery. Fortunately, many alternative treatments, with potentially different mechanisms of action, exist that are either currently not included in guidelines or are only recommended for use in specialist services - we refer to these as 'nonstandard'. These interventions are characterised by having a more limited evidence base to support their use, being associated with greater risks or invasiveness, and/or being more costly than standard treatments. The important clinical question is when to consider them for a particular patient.

\section{Problems and possible solutions}

The answer to the question is in some ways simple. If the probability of responding to (or tolerating) a further standard treatment is very low then a case can be made for using a non-standard treatment. However, the evidence base from which to draw such conclusions is far from ideal. Studies vary in their definitions of degree of treatment resistance necessary for inclusion. For example, the data supporting the use of quetiapine augmentation of antidepressants was obtained in studies of patients who had had a suboptimal response to their first, or possibly second, antidepressant. ${ }^{5}$ Inclusion in some studies is defined as much by chronicity as lack of treatment response. ${ }^{6}$ What studies there are tend to relate to patients who are treatment non-responsive rather than partially responsive, or to patients who have strong preferences for some treatments but not others. ${ }^{7}$ Overall, it is also important to note, there is little robust evidence from prospective sequential randomised controlled trials (RCTs) for the efficacy of options beyond third or fourth sequential treatments. ${ }^{8}$

One possible solution is to consider non-standard interventions only when all standard ones have been tried and failed. However, adequate trials of all possible combinations of pharmacotherapy alone would take literally decades. The evidence base does not support this strategy. Naturalistic data suggests that the response rate of patients with chronic treatment- resistant depression to standard interventions is only around $10 \%$ over 1 year. ${ }^{6}$ Further evidence suggests that the duration of untreated depression has a negative impact on eventual response. ${ }^{3}$ It is therefore clinically questionable to cycle endlessly through pharmacologically similar strategies. ${ }^{9}$

An alternative option is to use a 'threshold' approach. Conway and colleagues proposed treatment- resistant depression be divided into 'stage I' defined as failure of two interventions (medication or 
psychotherapy) and 'stage II' defined as failure of three interventions. ${ }^{9}$ They propose that less invasive non-standard interventions could be considered for stage I treatment- resistant depression and more invasive interventions (such as vagal nerve stimulation (VNS) or deep brain stimulation (DBS)) for stage II treatmentresistant depression. ${ }^{9}$ This threshold would allow for DBS for a patient after failure of 20 [th]mg of fluoxetine and 20 [th]mg of citalopram, both prescribed over 6 weeks, and 8 sessions of cognitivebehavioural therapy (CBT). We believe this is premature. This also illustrates the problem of a simple threshold approach with the challenge being how to find the best balance between timely access to alternative treatments $v$. unnecessary exposure to risky/ expensive interventions. The threshold for different interventions is also likely to be different with, for example, the threshold for ablative neurosurgery being substantially higher than for a welltolerated and safe non-standard medication. There are also a myriad of patient and illness factors that will also influence when a particular treatment might be deemed appropriate. Rather than a simple threshold we suggest a more nuanced approach with a defined reference point, which we refer to as 'multitherapy-resistant MDD' (MTR-MDD), to help guide clinicians, patients and commissioners.

\section{MTR-MDD}

The criteria proposed for MTR-MDD (outlined in the Appendix), for the reasons outlined above, are based more on consensus than clearcut evidence. They have been developed by clinicians drawn from primary care, secondary care and specialist services (see online supplement DS1, available at https://doi.org/10.1192/bjp.2017.33), including individuals with personal experience of MDD. The intention is that the MTR-MDD criteria can be broken down into its various component parts to help guide discussions between clinicians, patients and commissioners about when it may be reasonable to consider non-standard intervention. In totality, the criteria provide something of a 'back stop' - if a patient meets all of the criteria but has not been considered for non-standard treatment, then the question 'why not?' should be asked. We do not posit that MTR-MDD defines a specific subgroup of patients with MDD that may be characterised, for example, by a specific biology. However, the categorisation is potentially of use in research for stratification in trials of patients with particularly difficult to treat illness.

The term MTR-MDD has been chosen to reflect that: several interventions (more than two) must have failed to produce or maintain a response, or have been intolerable; non-pharmacological interventions should also have been tried and been ineffective or intolerable; and that the criteria are specific to MDD. The full MTR-MDD criteria are met if there has been 'a failure to respond, achieve remission, maintain a response/remission or tolerate' all the treatments listed in the Appendix. For many patients, it can be difficult distinguishing discrete episodes. It is inappropriate to re-trial medications that have failed in previous episodes unless the patient describes previous non-adherence and willingness to retry. As a result, these failures will usually be defined over the lifetime of the patient.

As in any area of medicine, non-response to an intervention should lead to a reappraisal of the diagnosis. ${ }^{10}$ Alternative primary diagnoses such as bipolar disorder should be carefully excluded. Comorbidities that may be contributing to the resistant nature of the MDD, such as substance use disorders, personality disorders, anxiety disorder and attention-deficit hyperactivity disorder (ADHD), should be identified and treated vigorously. Similarly, psychosocial factors should be addressed where possible. However, the presence of such comorbidity does not negate a patient meeting the criteria and hence consideration being given to non-standard interventions.

For all medications, an adequate trial is one where the clinician is confident (based on clinical judgement and patient history) that the patient has been adherent to a maximum licensed, or maximum-tolerated, dose for an adequate period of time. In the case of a maximum-tolerated dose, this must be a dose equivalent or higher than the generally regarded minimal therapeutic dose (for example as defined in the drug license). If a patient is not able to tolerate a minimum therapeutic dose, then the trial would be deemed to have failed on the basis of intolerance.

First- and second-line treatment trial durations are recommended to be 4-6 weeks, but for patients with resistant depression longer trials are recommended. ${ }^{1,2} \mathrm{We}$ recommend that at least one, and ideally two trials of antidepressants have been used for a minimum duration of 8 weeks at maximum or maximum-tolerated doses based on the need to balance 'efficiency' of a trial (shortest reasonable time) with the need to confidently exclude a potential response. The duration of augmentation trials is rarely described in guidelines. RCT data suggests a relatively rapid response to some agents, for example antipsychotic augmentation (such as quetiapine ${ }^{5}$ ), whereas other strategies may take longer at least in part because of the time needed to reach a therapeutic level (such as lithium). Nevertheless, we would recommend a minimum of 4 weeks, and ideally 8 weeks, at a therapeutic dose, for augmentation trials.

With regards to non-tolerance, in almost all circumstances, clinicians should endeavour to establish that all reasonable efforts have been taken to ensure that the patient can tolerate at least the minimum therapeutic dose. This may require extended dose titration periods, using preparations that allow starting at very low doses, the use of other medications (such as benzodiazepines for example when using an activating drug such as aripiprazole) and frequent review. In many situations, the clinician should recommend at least one other drug from the same drug class to ensure that intolerance is class- rather than drug-specific.

Determining the adequacy of a course of psychotherapy may be more difficult, as factors not always directly related to the patient or their illness have been demonstrated to have an impact on outcome, including therapeutic alliance, therapist adherence to the therapeutic modality and match between patient and therapeutic modality. ${ }^{11-13}$ Some patients need several months of preparation by clinicians or psychotherapists to develop the psychological mindedness to benefit from a course of any psychotherapy. These considerations and assessment of past psychotherapy may of itself be a justification for seeking an expert psychotherapy opinion.

The rationale for including electroconvulsive therapy (ECT) as a requirement in the MTR-MDD criteria is that ECT is, for most people, a choice that offers a high chance of improvement ${ }^{14}$ and acceptable levels of risk compared with more advanced and/or less evidence-based options. However, a failure of ECT to lead to a maintained response despite antidepressant prophylaxis, or a refusal/inability to undergo a trial of ECT, would be an appropriate prompt to consider alternative interventions with patients, especially if they meet MTR-MDD criteria.

\section{Using the MTR-MDD criteria to guide the use of non- standard treatments}

Non-standard interventions can be considered to exist on a spectrum. At one end, there are those that have a relatively strong evidence base to support their use in MDD, are easy to implement, well tolerated, non-invasive and relatively cheap. At the other end, interventions have more limited evidence to support them, their 
use is more complex to undertake, they are associated with more risks or invasiveness and/or they are of higher costs than standard interventions. The position of an intervention on the spectrum also depends on its regional and national availability and the expertise of the clinician(s) using it.

Infrequently used interventions are more likely to be appropriate for specialised centres. This, as well as other factors, is prone to change over time as more evidence is acquired. Consideration of these issues can help decisions about the potential position in the treatment algorithm of specific non-standard interventions. Interventions supported by RCT data, well tolerated and of a similar cost to standard treatments are likely to be used early on. It may well be totally inappropriate to wait until a patient has a duration of illness of 2 years and/or has had three episodes of illness. When such interventions are used will be determined primarily by clinician expertise and local availability.

The threshold for some non-standard interventions may be determined by individual elements of the MTR-MDD criteria. For example, transdermal selegiline has been shown to be particularly effective in patients with atypical depression, ${ }^{15}$ but in many parts of the world is extremely expensive to acquire. As a result, it is probably reasonable that the MTR-MDD antidepressant criteria are met, including that a standard monoamine oxidase inhibitor (MAOI) that irreversibly blocks monoamine oxidase $\mathrm{B}$ (MAO-B) is tried first. Similarly, the complex psychotherapy cognitive-behavioural analysis system of psychotherapy (CBASP), which has data supporting its use in treatment-resistant $\mathrm{MDD}^{16}$ but which is non-standard primarily because of limited availability, is likely to only be considered if a patient meets the MTR-MDD psychotherapy criteria.

Modafinil is not included in NICE depression guidelines, ${ }^{1}$ although it is mentioned as an option for use in specialist centres in BAP guidelines ${ }^{2}$ on the basis of four RCTs conducted primarily in patients with non-treatment-resistant MDD. ${ }^{17}$ In general it is well tolerated and can be combined with most antidepressants. It is therefore at the more benign end of the non-standard therapy spectra. It would seem reasonable to consider this if the MTR-MDD criteria for pharmacological augmentation are met. Conversely, pramipexole, which is supported by just two conflicting $\mathrm{RCTs}^{18}$ and is more complex to use (because of the potential for impulse control disorders such as gambling ${ }^{19}$ is likely to be considered only after a range of interventions have been unsuccessful i.e. beyond MTR-MDD augmentation criteria. Further along the spectra, intravenous ketamine, which is supported by a number of studies but which is also associated with risk, invasiveness and limited duration of effect ${ }^{20}$ is likely to only be used if broader MTR-MDD criteria are met.

There are an increasing number of non-drug physical treatments for MDD that vary in their degree of invasiveness. Transcranial magnetic stimulation (TMS) is well tolerated and supported by NICE recommendations. ${ }^{21}$ Hence by our definitions it is a 'standard' intervention although its use is limited by availability. It may be considered before ECT in the treatment algorithm for some patients at less immediate risk. Conversely, VNS has been reviewed by NICE and recommended for use only 'with special arrangements for clinical governance, consent and audit or research'. ${ }^{22}$ It might be appropriate to consider this if ECT has been considered inappropriate, unacceptable or it has led to an inadequate response. Given its cost and limited availability it is also likely that patients will meet the other MTR-MDD criteria. The full MTR-MDD criteria are likely to be the bare minimum for consideration of anterior cingulotomy or other neurosurgical procedures, although the concern is that even for this highly invasive irreversible intervention there is often currently too long a delay before a patient is considered for this.

Clearly an additional factor that influences where an intervention is placed in the algorithm is the patient. For example, a concern about medication may lead to the use of a non-invasive relatively cheap non-drug physical intervention such as TMS early on. Alternatively, the clinical characteristics of a patient may influence choice. With regards to standard interventions an example would be the use of ECT early on for patients with psychotic depression or marked psychomotor retardation. Similarly, VNS might be considered for patients with highly recurrent depression despite ongoing prophylactic medication. ${ }^{23}$

\section{Discussion}

With the welcome burgeoning of novel non-standard interventions, the question is where in the treatment algorithm they should be used. The appropriate place will vary between patients and interventions. Clinical decisions regarding this are a complex interplay between various factors such as: the patient's clinical state; research evidence; patient preference and expectations; and the expertise and experience of the clinician. A significant concern is that non-standard interventions are only brought into consideration much later than might be appropriate and that this is to the detriment of the patient, although they may also be used inappropriately early in some situations. We argue that the framework and MTR-MDD criteria described in this paper should complement clinical expertise rather than replace it, to act as a reference point around which to gauge when it is clinically appropriate to use non-standard interventions. We believe that a patient meeting all of the MTR-MDD criteria should be considered for non-standard treatment if this has not already happened. The criteria also act as a prompt for clinicians with regards standard interventions. Whether MTR-MDD criteria are appropriate for all age groups, including adolescents, is an open question. At the very least we hope that this analysis piece prompts debate around these issues.

R. H. McAllister-Williams, PhD, MD, FRCPsych, Institute of Neuroscience, Newcastle University, Newcastle upon Tyne and Regional Affective Disorders Service, Northumberland Tyne and Wear NHS Foundation Trust, Newcastle upon Tyne:

D. M. B. Christmas, MD, MRCPsych, Advanced Interventions Service, Ninewells Hospital \& Medical School, Dundee; A. J. Cleare, FRCPsych, Centre for Affective Disorders, Institute of Psychiatry, Psychology and Neuroscience, King's College London, Disorders, Institute of Psychiatry, Psychology and Neuroscience, King's College Lon
London and Maudsley NHS Foundation Trust, London; A. Currie, MBChB, MPhil, FRCPsych, Regional Affective Disorders Service, Northumberland Tyne and Wear NHS Foundation Trust, Newcastle upon Tyne; J. Gledhill, MBBS, MRCGP, North Durham Clinical Commissioning Group, County Durham; L. Insole, MRCPsych, North East Community Mental Health Team, Northumberland Tyne and Wear NHS Foundation Trust, Newcastle upon Tyne; A. L. Malizia, MD, MRCPsych, Neuropsychopharmacology and Neuromodulation, Rosa Burden Centre, Southmead Hospital, North Bristol NHS Trust, Bristol; M. McGeever, MBBS, MRCGP, DRCOG, DFSRH, Benfield Park Medical Group, Newcastle Gateshead Clinical Commissioning Group, Newcastle upon Tyne; R. Morriss, FRCPsych, Centre for Mood Disorders, Institute of Mental Health, University of Nottingham, Nottingham; L. J. Robinson, PhD, DClinPsy, Institute of Neuroscience, Newcastle University, Newcastle upon Tyne and Regional Affective Disorders Service, Northumberland Tyne and Wear NHS Foundation Trust, Newcastle upon Tyne; M. Scott, MBBS, MRCGP, Newburn Surgery, Newcastle Gateshead Clinical Commissioning Group, Newcastle upon Tyne; P. R. A. Stokes, PhD, FRCPsych, Centre for Affective Disorders, Institute of Psychiatry, Psychology and Neuroscience, King's College London, London and Maudsley NHS Foundation Trust, London; P. S. Talbot, MD, MRCPsych, Wolfson Molecular Imaging Centre, University of Manchester and Specialist Service for Affective Disorders, Greater Manchester Mental Health NHS Foundation Trust, Manchester; A. H. Young, PhD, FRCPsych, Centre for Affective Disorders, Institute of Psychiatry, Psychology and Neuroscience, King's College London, London and South London and Maudsley NHS Foundation Trust, London.

Correspondence: R. H. McAllister-Williams, Wolfson Research Centre, Campus for Ageing and Vitality, Newcastle upon Tyne, NE4 5LP, UK. Email: r.h.mcallister-williams@ncl.ac.uk

First received 15 Aug 2016, final revision 1 Aug 2017, accepted 17 Aug 2017

\section{Supplementary material}

Supplementary material is available online at https://doi.org/10.1192/bjp.2017.33. 


\section{Funding}

This paper arose out of an advisory board meeting convened in November 2015 by LivaNova, the manufacturers of VNS therapy. Several of the named authors (R.H.M.W., D.M.B.C. A.J.C. A.L.M., P.R.A.S., P.S.T., A.H.Y.) attended this meeting and were paid an honorarium by LivaNova. The paper has been conceived, written and submitted for publication entirely independently of LivaNova and the authors have not been remunerated for writing it.

This report represents independent work in part funded by the National Institute for Health Research (NIHR) Biomedical Research Centres at South London and Maudsley NHS Foundation rship in Applied Health Research and Care East Midlands (CLAHRC EM) and MindTech Mental Health Technology Co-operative, Nottingham. The views expressed are those of the authors and not necessarily those of the NHS, the NIHR, or the Department of Health.

\section{Acknowledgements}

The authors would like to thank two anonymous reviewers of previous versions of this paper. Their thoughtful comments provoked a significant re-conceptualisation of the MTR-MDD criteria.

\section{Appendix}

\section{Proposed criteria for MTR-MDD}

The patient: diagnosed with MDD (using (DSM-5). ${ }^{24}$

Their depression: MDD of at least moderate severity.

Their treatment

(a) Psychotherapy. At least two trials of structured, evidence-supported psychological therapy. ${ }^{1}$ The trials should ideally each be of a different modality and provided by a different therapist. In both cases, the clinician should assure themselves that the patient has received a structured course of therapy delivered by an experienced therapist with whom the patient had a good therapeutic relationship. Ideally, at least one of the trials should have been of at least 16 hours duration and at least one trial should have been given in combination with pharmacotherapy.

(b) Antidepressants. Four adequate trials of antidepressants. There is little consensus with regards how antidepressants should be divided into different 'classes' and how important it is that drugs from different classes are trialed. However, it is recommended that the trials should not all be from the same class of drugs and that at least two trials are using antidepressants that are viewed as being potentially more efficacious in severe depression and/or compared with other antidepressants, for example as listed by BAP guidelines (clomipramine, venlafaxine $(\geq 150[\mathrm{th}] \mathrm{mg})$, escitalopram (20[th]mg), sertraline, amitriptyline or mirtazapine). ${ }^{2}$ We would also recommend consideration of a traditional MAOI (for example phenelzine), especially for patients with atypical symptoms.

(c) Pharmacological augmentation. At least two adequate trials of an evidence-based augmentation/combination agent given in combination with an antidepressant. Ideally these should both be agents listed as first-line options in BAP guidelines (lithium (ideally with a plasma level of $0.6-1.0[\mathrm{th}] \mathrm{mmol} / \mathrm{L}$ ), quetiapine and aripiprazole). ${ }^{2}$

(d) ECT. A trial of ECT (at least eight treatments, and ideally bilateral if tolerated).

For all treatments: the requirement for a treatment may be waived if there is a recognised contraindication or the patient has, despite extensive discussions and the provision of information, declined it, or there have been well-documented adverse effects that have limited tolerability. This applies to ECT, psychotherapy and medication.

Given evidence for possible greater efficacy of a structured psychological treatment in combination with medication, ${ }^{25,26}$ a period of combined treatment, possibly over a period of 9-15 months, is recommended.

\section{References}

1 National Institute for Health and Care Excellence. Depression: The Treatment and Management of Depression in Adults (update). Clinical Guideline 91. NICE, 2009

2 Cleare A, Pariante CM, Young AH, Anderson IM, Christmas D, Cowen PJ, et al. Evidence-based guidelines for treating depressive disorders with antidepressants: a revision of the 2008 British Association for Psychopharmacology guidelines. J Psychopharmacol 2015; 29: 459-525.

3 de Diego-Adelino J, Portella MJ, Puigdemont D, Perez-Egea R, Alvarez E, Perez V. A short duration of untreated illness (DUI) improves response outcomes in first-depressive episodes. J Affect Disord 2010; 120: 221-5.

4 Rhebergen D, Beekman AT, De GR, Nolen WA, Spijker J, Hoogendijk WJ et al. Trajectories of recovery of social and physical functioning in major depression, dysthymic disorder and double depression: a 3-year follow-up. J Affect Disord 2010; 124: 148-56.

5 Bauer M, Pretorius HW, Constant EL, Earley WR, Szamosi J, Brecher M. Extended-release quetiapine as adjunct to an antidepressant in patients with major depressive disorder: results of a randomized, placebo-controlled, double-blind study. J Clin Psychiatry 2009; 70: 540-9.

6 Dunner DL, Rush AJ, Russell JM, Burke M, Woodard S, Wingard P, Allen J. Prospective, long-term, multicenter study of the naturalistic outcomes of patients with treatment-resistant depression. J Clin Psychiatry 2006; 67: 68895.

7 Dunlop BW, Kelley ME, Aponte-Rivera V, Mletzko-Crowe T, Kinkead B, Ritchie JC, et al. Effects of Patient Preferences on Outcomes in the Predictors of Remission in Depression to Individual and Combined Treatments (PReDICT) Study. Am J Psychiatry 2017; 174: 546-56.

8 Gaynes BN, Warden D, Trivedi MH, Wisniewski SR, Fava M, Rush AJ. What did STAR*D teach us? Results from a large-scale, practical, clinical trial for patients with depression. Psychiatr Serv 2009; 60: 1439-45.

9 Conway CR, George MS, Sackeim HA. Toward an evidence-based, operational definition of treatment-resistant depression: when enough is enough. JAMA Psychiatry 2017; 74: 9-10.

10 Haddad PM, Talbot PS, Anderson IM, McAllister-Williams RH. Managing inadequate antidepressant response in depressive illness. Br Med Bull 2015; 115: 183-201.

11 Arnow BA, Steidtmann D, Blasey C, Manber R, Constantino MJ, Klein DN, et al The relationship between the therapeutic alliance and treatment outcome in two distinct psychotherapies for chronic depression. J Consult Clin Psychol 2013; 81: 627-38.

12 DeRubeis RJ, Cohen ZD, Forand NR, Fournier JC, Gelfand LA, Lorenzo-Luaces L. The Personalized Advantage Index: translating research on prediction into individualized treatment recommendations. A demonstration. PLoS One 2014; 9: e83875

13 Weck F, Grikscheit F, Jakob M, Hofling V, Stangier U. Treatment failure in cognitive-behavioural therapy: therapeutic alliance as a precondition for an adherent and competent implementation of techniques. Br J Clin Psychol 2015; 54: 91-108.

14 Scottish ECT Audit Network. Annual Report 2015: A Summary of ECT in Scotland for 2014. Scottish ECT Audit Network, 2015.

15 Pae CU, Lim HK, Han C, Neena A, Lee C, Patkar AA. Selegiline transdermal system: current awareness and promise. Prog Neuropsychopharmacol Biol Psychiatry 2007; 31: 1153-63.

16 Wiersma JE, Van Schaik DJ, Hoogendorn AW, Dekker JJ, Van HL, Schoevers RA et al. The effectiveness of the cognitive behavioral analysis system of psychotherapy for chronic depression: a randomized controlled trial. Psychother Psychosom 2014; 83: 263-69.

17 Goss AJ, Kaser M, Costafreda SG, Sahakian BJ, Fu CH. Modafinil augmentation therapy in unipolar and bipolar depression: a systematic review and metaanalysis of randomized controlled trials. J Clin Psychiatry 2013; 74: 1101-7.

18 Kleeblatt J, Betzler F, Kilarski LL, Bschor T, Kohler S. Efficacy of off-label aug mentation in unipolar depression: a systematic review of the evidence. Eur Neuropsychopharmacol 2017; 27: 423-41.

19 Strejilevich SA, Martino DJ, Igoa A, Manes F. Pathological gambling in a bipolar patient treated with pramipexole. J Neuropsychiatry Clin Neurosci 2011; 23: E2-3.

20 Bobo WV, Voort JL, Croarkin PE, Leung JG, Tye SJ, Frye MA. Ketamine for treatment-resistant unipolar and bipolar major depression: critical review and implications for clinical practice. Depress Anxiety 2016; 33: 698-710. 
21 National Institute for Health and Care Excellence. Repetitive Transcranial Magnetic Stimulation for Depression. NICE Interventional Procedure Guidance (IPG542) NICE, 2015

22 National Institute for Health and Care Excellence. Vagus Nerve Stimulation for Treatment Resistent Depression. NICE Interventional Procedure Guidance (IPG 330). NICE: 2009.

23 Aaronson ST, Sears P, Ruvuna F, Bunker M, Conway CR, Dougherty DD, et al. A 5-year observational study of patients with treatment-resistant

depression treated with vagus nerve stimulation or treatment as usual: comparison of response, remission, and suicidality. Am J Psychiatry 2017; 174 640-8.
24 American Psychiatric Association. Diagnostic and Statistical Manual of Mental Disorder (5th edn) (DSM-5). APA, 2013

25 Cuijpers P, Berking M, Andersson G, Quigley L, Kleiboer A, Dobson KS. A metaanalysis of cognitive-behavioural therapy for adult depression, alone and in comparison with other treatments. Can J Psychiatry 2013; 58: 376-85.

26 Morriss R, Garland A, Nixon N, Guo B, James M, Kaylor-Hughes C, et al. Efficacy and cost-effectiveness of a specialist depression service versus usual specialist mental health care to manage persistent depression: a randomised controlled trial. Lancet Psychiatry 2016; 3: 821-31. 